\title{
L'Usine-Barrage de Belle-Isle-en-Terre (Côtes-du-Nord)
}

C'est le $1^{\text {er }}$ septembre 1923 que M. Le Trocquer, Ministre des' Travaux-Publics, a inaugurè l'usine-barrage hydro-électrique de. Belle-Isle-en-Terre construite par la Socièté Technique et Industrielle d'entreprises, d'après la conception et sous la direction de M. E. Froté, Ingénieur, pour les Papeteries Vallée \& Cie.

Cette usine qui forme avec le barrage un seul monolithe en béton armé est la première de ce genre en Europe.

Il s'agissait de barrer la vallée de la rivière Le Leguer dans sa partie la plus rétrécie à 600 mètres environ en amont du pont de la route de Plouaret à Bégard, par la construction d'un barrage pour utiliser le dénivellement de la rivière de $13 \mathrm{~m} .50$ qui existe entre cet endroit et la sortie du canal de décharge de l'ancienne usine hydro-électrique des Papeteries, à Belle-Isle-en-Terre.

Ce barrage a ainsi créé un lac de 3.100 mètres de longueur avec une superficie de 26 hectares et une chute utilisable de $13 \mathrm{~m} .30$.

L'auteur du projet pour éviter toute perte de charge d'un canal d'amenée ou d'une conduile a conçu un barrage en béton armé, sous lequel sont aménagés les locaux recevant toutes les machines et appareils d'une usine hỵdro-électrique,

Débit. - D'après les jaugeages et relevés journaliers au limimètre de l'usine de la papeteric, exécutés depuis de longues années, le débit moyen utilisable se monte à $2,8 \mathrm{~m}^{3} / \mathrm{sec}$. pendant les six mois d'été, tandis qu'il se monte à $6 \mathrm{~m}^{3} / \mathrm{sec}$. pendanl la période d'hiver. En se basant sur le débit de la période hivernale, le lac créé permettant d'accumuler les eaux pendant les heures dc faible consommation pour les utiliser pendant les heures de forte
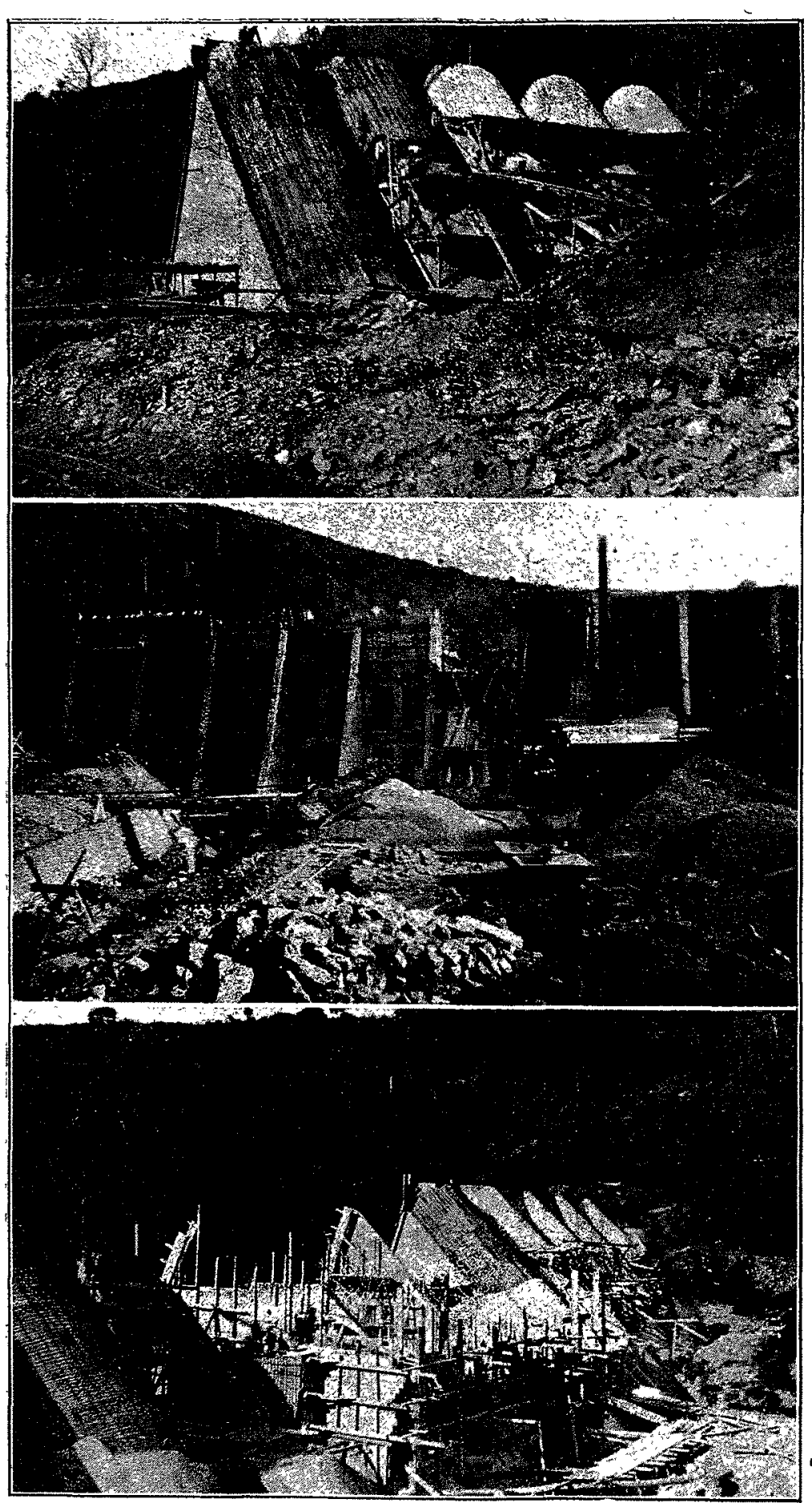

Construclion de l'Usine de Belle-Isle-en-Terre. aux axes turbines est de $1.350 \mathrm{HP}$. La variation des débits entre les périodes d'hiver et d'été étant très sensible il a été admis une unité de $220 \mathrm{HP}$, une seconde de $290 \mathrm{HP}$ et une troisième de 840 FIP de façon à éviter les trop grandes pertes de charge.

Barrage. - Quinze contreforts de hauteurs différentes, en béton armé de $0,20 \mathrm{~m}$. d'épaisseur, sont placés à $4 \mathrm{~m}$. 86 de distance d'axe en axe parallèlement au cours de la rivière. La plus grande hauleur est de $16.35 \mathrm{~m}$.

Ces contreforts supportent des voûtes en béton armé inclinées à $45^{\circ}$ qui relienment l'au du lac et transmettent les pressions provenant des eaux du lac, el du poids mort de l'ouvrage perpendiculairement sur le roc graniteux par des semelles en béton armé formant patin le long de la base. A cet cffet le nu aval de ces contreforls a une inclinaison de $60^{\circ}$ par rapport à l'horizontale pour ceux qui sont pleins et de $50^{\circ}$ pour les deux qui traversent la salle des machines, $c$ ayant par conséquenl de grandes ouvertures.

La longueur des semelles est de $0 \mathrm{~m} .95$ de façon à ce que la pression sur le roc ne dépasse nulle part $5,5 \mathrm{~kg}$ par $\mathrm{cm}^{2}$.

Pour reculer diune part le. centre de gravité du contrefort vers l'aval, el diminuer de ce fait l'excentricité de la résullante des efforts, et pour donner d'autre part un aspect plus approprié à l'ensemble du barrage à l'aval, l'épaisscur des contreforts a élé portée aux extrémilés au double de l'épaisseur ordinaire, c'est-à-dire à $0 \mathrm{~m} .10$.

L.es contreforts sont armés par un double quadrillage de barres d'acier rondes de $7,5 \mathrm{~m} / \mathrm{m}$ de diamètre.

Des renforcements considéraconsommation l'usine a été établie pour un lébil de $10 \mathrm{~m} \mathrm{~m}^{3} / \mathrm{sec}$.

Il est à remarquer que les plus forles crues peuvent alleindre un débit de 40 à $50 \mathrm{~m}^{3} / \mathrm{sec}$.

Chute. - Le niveau de la retenue ayant été fixé à la cole 13 mètres 27 et le niveau de la rivière canalisée en aval du barrage étant à la cote - 0,05 m., la chute utilisable est de $13 \mathrm{~m}$. 32. En cas de crues extraordinaires elle peut se réduire à 11 mètres environ.

Force. - En prenant un débit de $10 \mathrm{~m}^{3} / \mathrm{sec}$. la force disponible bles ont été exéculés dans les contreforts ayant des ouvertures pour l'amenagement des salles des machines et d'appareillage. Il y a à considérer les renforcements nécessités pour la transmission de la réaction des voutes sur les parties latérales des ouvertures, el ccux nécessités pour l'oblention d'une fondation homogène el d'une répartilion linéaire de la réaclion sur loute la longueur de la base du contrefort.

Dans ce but, il a été élabli, dans le contrefort même, de véritables poutres, dont la portée englobe, dans la direction de la pression, toutes les ouvertures voisines. 
Pour être absolument sûr, il n'a élé admis dans les calculs comme laateur de poutre que la distance entre le nu supérieur de l'ouverture el la ligne d'intersection extérieure des deux voûtes consécutives, sans tenir ainsi comple des voûtes elles-mêmes qui font cependant partie intégrante de ces poutres.

L'entrecroisement longitudinal est assuré par la passerclle supérieure, par une série d'entreprises de $0,20 \times 0,20$, dispošées entre les contreforts et par les planchers de l'usine.

Ja passerelle qui se trouve à $1 \mathrm{~m} .50$ au-dessus du niveau de la retenue permel de passer d'une rive à l'autre et d'accéder à la passerelle des mancuvres des vannes des lurbines.

Les voûtes inclinées à $45^{\circ}$, d'une épaisseur variant de $0 \mathrm{~m}$. 18 à $0 \mathrm{~m} .12$ en béton armé d'un double quadrillage de barres d'acier, sont de forme semi-circulaire de $2 \mathrm{~m} .33$ de rayon intérieur suivant la coupe horizontale. Toutes les précautions ont élé prises, et un procédè spécial a été adopté pour oblenir dès le début des voûtes absolument élanches, car dles devaient relenir, d'une parl, l'eau du lac, et abriter les machines et les appareils, d'autre part.

Ĺnine. - Les salles destinées a lemplacement des machines et des appareils sont aménagées an milieu du barrage au-dessus du lit de la rivière, el occupent lespace situé entre cinq contreforts consécutifs.

I'n mur vertical en bélon armé construit entre les cing contreforts, au-dessous"de la passerelle supéricure du bairrage, ferme cel espace du côlé aval. I)es fenêtres placées dans ce mur éclairent largement les salles.

L'espace entre les cinq contreforts forme qualre compartiments el est divisé en trois élages superposés.

Le plancher, ainsi que la paroi du côté du lac, du premier étage, sont constilués par le gros bélon qui entoure les conduiles d'aspiration des turbines. L'espace entre ce plancher et le dessous

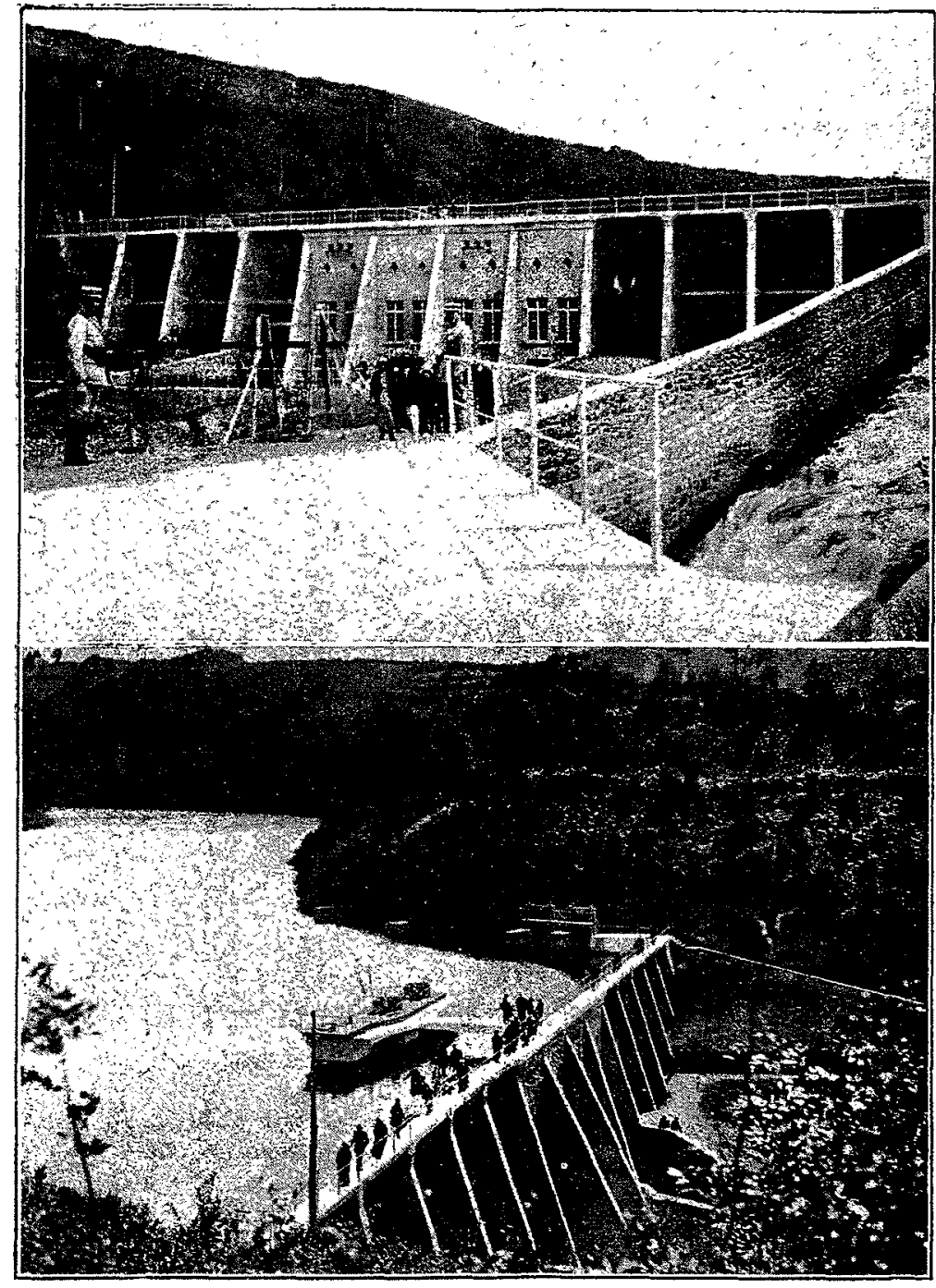

Vue de lusine et du Barrage de Belle-Isle-en-Terre, la construction terminćc.
La porte d'entrée s'ouvre sur le premier compartiment qui sert de local de déchargement et de réparation.

Une trappe mobile fixée dans le plancher permet de descendre les transformateurs au premier étage. Le service du personnel se fait par un escalier entre le premier et le deuxième, et par une échelle entre le deuxième et le troisième étage. Dans ce premier compartiment se trouve aussi la commande de l'une des deux vannes de fond.

Pour facililer la communication entre les trois groupes turboalternateurs el pour avoir une vue d'ensemble depuis le tableau pupitre qui se trouve vis-à-vis du groupe médian, de grandes ouvertures sont ménagées dans les deux contreforts du milieu qui ont été sérieusement renforcés à cet effet, comme cela a élé mentionné précédemment.

La chambre de chacue turbine est placée dans la voûte correspondante. Elle communique d'une part avec le lac par une grande ouverture pouvant être fermée par une vanne, devant laquelle se trouve une grille mobile, et d'autre part avec l'extérieur par une cheminée verticale rectangulaire, dans laquelle glisse la vanne de fermeture.

Une petite vanne cylindrique auxiliaire est placée à mi-hauteur de la cheminée pour y introduire l'eau du lac et faciliter ainsi le levage de la vanne d'entrée. Un clapet permet la vidange de la chambre de la turbine par le tuyau d'aspiration.

Les trois cheminées aboulissent, en haut, à une passerelle secondaire, sur laquelle reposent les appareils de levage des vamnes et grilles des chambres des lurbines.

Cette passerelle est parallile à celle de la crète du barrage, et établie au mème niveau. Elles sont reliées ensemble par une troisième passerelle.

L'ensemble des différents planchers et parois de l'usine, des chambres des turbines et des che- de la passerelle supérieure du barrage est divisé en trois élages superposés par deux planchers inlermédiaires en bélon armé, qui suivenl, en plan, la forme des voûtes.

Trois compartiments du premier élage de $3 \mathrm{~m} .40$ de hauteur, qui communiquent ensemble par deux ouverlures daus chaque contrefort sont réservés aux transformaleurs el aux càbles de connexion, ceux du second élage de $4 \mathrm{~m}$. de hauteur au lableau pupitre et aux trois groupes lurbo-alternateurs, chacun d'eux étant placé daus un des compartiments, et coux du troisième élage de 4 m. 50 de haulcur, aux parafoudres et aux départs des lignes.

La porle d'entrée de l'usine se lrouve au second étage et est aménagée dans le contrcfort de la rive gauche formant pignon de l'usine.

L'accès se fait au moyen d'un ter're-plein en remblai où aboutit le chemin d'accès. minces, représenle un monolithe en béton armé extrèmement incrle dans lous les sens de sorte que les vibrations des turbines et allernateurs n'ont qu'un très faible effet sur l'ensemble de l'ouvrage.

Cet effet est pour ainsi dire réduit à néant, du fait que chaque turbine repose sur le massif de béton qui entoure sa conduite d'aspiration, dont il est parlé plus haut, et qui repose directement sur le rocher entre les contreforts.

Les chambres des trubines avec leur cheminée ainsi que les conduites d'aspiration sonl de différentes dimensions, correspondant aux puissances des trois turbines qui sont respeclivement de 220, 290 et 840 chevaux.

Les eaux sortant des conduites d'aspiration se réunissent dans un bassin, dont le radier correspond au fond du canal de fuite. C'est le lit même de la rivière qui a été approfondi et canalisé 
par deux murs latéraux sur unc longueur d'environ 100 mètres, qui sert de camal de fuite.

Pour éviter, dans la salle des alternateurs, loute condensation le long des voûtes, une seconde voûte a été établie à une pelite distance de la première pour créer un matelas d'air servant d'isolant. Pour y assurer une circulation d'air, le plancher supérieur de la salle des parafoudres a été interrompu partiellement. A sa partie inférieure, un caniveau, légèrement incliné, permet l'évacuation des eaux de condensation éventuelles par les contrefortspignons de l'usine.

Vannes de fond. - Deux vamnes de fond ont été prévues pour l'évacuation des eaux pendant la construction du barrage ef pour la vidange éventuelle du lac dans la suite. L'une est placée sur la rive gauche de la rivière, entre les contreforts du premier compartiment de l'usine, et l'autre sur la rive droite en dehors de l'usine, entre son dernier contrefort et le suivant.

La première est circulaire, de $1 \mathrm{~m} .20$ de diamètre et permet l'évacuation des eaux dans le canal de fuite par un Luyau en bėton armé, traversant le fond de l'usine.

Cette vanne est mancuvrée depuis l'intérieur de l'usine.

La seconde vanne de fond est rectangulaire et est placée sur le plan incliné de la voûte. Elle est manœuvrée depuis la passerelle supérieure du barrage et permet la vidange du lac par un canal, construit entre deux contreforts, qui se déverse après un parcours de 40 mètres dans le canal de fuite.

Déversoir. - Pour évacuer les plus grandes crues, qui sont estimèes à $50 \mathrm{~m}^{3}$ par seconde, il a été construit un déversoir fixe de 50 mètres de longueur en deux ouvertures de 25 mètres chacune, et à son extrémité droite deux vannes de $2 \mathrm{~m} .65 \mathrm{de}$ largeur chacune, et de $1 \mathrm{~m} .85$ de hauteur. Ce déversoir, laillé dans le roc, sur la rive gauche, est disposé à peu près perpendiculairement au barrage.

Son mur, dont le seuil se trouve à $1 \mathrm{~m} .50$ en contre-bas de la crête du barrage, est construil de façon à pouvoir recevoir une surélévation mobile de $0 \mathrm{~m} .70$.

L'eau du déversoir est évacuée au moyen d'un canal de décharge, taillé à flanc de coleau en échelle dans le roc pour permettre aux poissons d'accéder au lac. Ce canal aboulil dans le lit de la rivière, à environ 120 mètres du barrage, et est franchi par le pont en béton armé du chemin d'accès de l'usine.

Machines. - Acluellement deux groupes Lurbo-alternateurs de 220 et de 290 chevaux, sonl en fonction, le troisième groupe de 840 chevaux, va être installé prochainement.

Les turbines sont accouplées directement aux alternateurs.

Les caractéristiques de la première Lurbine installée el fournie par la Maison Escher Wyss \& C ic se résument comme suit :

\begin{tabular}{|c|c|c|c|}
\hline Chute netle .......... & 10,80 & 13 & mèlres. \\
\hline 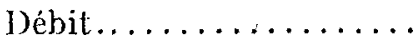 & 1520 & 1620 & $\mathrm{lil} / \mathrm{sec}$. \\
\hline Puissance...$\ldots \ldots \ldots$ & 173 & 220 & chevaux (IIP) \\
\hline Vitesse.............. & 600 & 600 & tours/min. \\
\hline Rendement pour $8 / 8 \mathrm{HP} .$. & 79 & 78,5 & $\%$ \\
\hline
\end{tabular}

De la puissance $7 / 8 \mathrm{HI}$...

Normale sous la $6 / 8 \mathrm{H} 1 \mathrm{l}^{3} .$.

Chute correspond. $4 / 8 \mathrm{HP}$.

$5 / 8 \mathrm{FP}$.

80,5

79

68

75

81
80
76,5
71

$\%$

$\%$

$\%$

$\%$

Ces rendements s'entendent avec une lolérance de $\pm 2 \%$ sous la chute de 10,80 el 13 mètres.

Le réglage est de :

Sous décharges brusques de ....... Augmentation de vitesse maxim...

$$
\begin{aligned}
& 72 \\
& 21 / 2
\end{aligned}
$$

$290 \mathrm{Hp}$
$16 \%$

Le rendement de la seconde turbine de 290 chevaux est identique à celui de la première.

Ces deux turbines sont du lype horizontal ouverles, simples, et chacune des deux machines actionne directement un altemateur de mème vitesse.

Le plancher de la salle des allernateurs ayant élé fixé à 5 m. 50 au-dessus des plus basses eaux, chaque turbine a dû c̀lre construite avec un tuyan daspiralion de béton armé noyé dans du gros béton, dont la haulcur entre l'arbre de la lurbine et le niveau de l'eau atteint $6 \mathrm{~m}, 45$.

Afin d'utiliser au mieux la chute, les turbines ont été construites avec une roue mobile d'un nouveau modèle, qui jossède un rendement excellent à vitesse constante pour toute la gamme des chutes de la retenue ainsi que pour les ouverlures du distributeur les plus variables.

La rone mobile des lurbines est intentionnellement calée en porte à faux sur l'extrémité do l'arbre, afin de laisser le coude de succion absolument libre.

Cette disposition gui facilite enormément le démontage favorise également le bon rendement, car les filets liquides sortant de la roue mobile s'écoulent librement, puisque le coude n'est traversé par aucun arbre.

Chaque turbine est munie d'un régulateur universel à pression d'huile, cet appareil possédant une grande sécurité de marche jointe à une haute sensibilité, agit sur les directrices mobiles par l'intermédiaire de leviers, anneau de réglage et bielletles.

Pour augmenter le PD2 de l'alternateur, chaque turbine possède un volant supplémentaire combiné avec l'accouplement rigide; le palier de la turbine el un palier à butées absorbant loute la poussée axiale de la roue mobile el sa sécuritế de marche est assurée par une circulation d'eau dans le bain d'huile.

La troisième turbine de 840 chevaux sera du meme type.

L'alternateur qui est accouplé directement à la première turbine est prévu pour les caractéristiques suivantes :

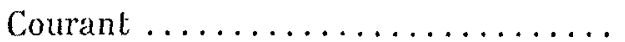

Nombre de paliers .............

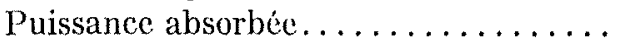

Puissance débitćc................

Tension. ...................

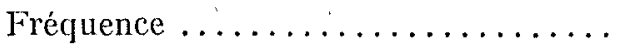

Vilesse....................

Vitesse d'emballement............

Excitation $\ldots \ldots \ldots \ldots \ldots \ldots \ldots \ldots$ triphasé. 2 -à flasques.

$238 \mathrm{HP}$.

$200 \mathrm{KV \Lambda}$.

220 volls.

50 périodes. 600 tours par minute. $1260-$ - -32 ampèr. 
L'alternatcur installé est du type à pòles alternés avec induil fixe el inducteur mobile excité par du courant continu provenant d'une excilatrice séparée.

La carcasse sur le pourlour de laquelle sont aménagés des orifices de ventilation est en une seule pièce. Le fer de l'induit se compose de tôles légèrement siliceuses, à pertes minimes; les encoches sont mi-fermées, l'isolation des rainures a été faite par des tubes sans joint en micanite qui y ont été logés avant de procéder au bobinage.

Les tètes des bobines sont serrées dans un bandage de toile isolante au compound el sont en outre imprégnées de laque. l.'s bobines d'extrémilé qui sont conncctées aux bornes de lallernateur, sont protégées spécialement contre les surtensions.

Les deux llasques, ouverles, venues de fonte avec les paliers, sonl fixées des deux côlés de la carcasse concentriquement à celle-ci, ce qui permet l'installation de la machine sans plaque de fondation el directement sur le massif. Les lètes de paliers el les coussinets sont en deux pièces el pourvus d'un graissage automatique à bagues el facilement contròlable pendant la marche.

I.arbre esten acier S. M. forgé el prolongé à ses deux extrémités : sur l'une d'elles est fixé le manchon pour laccouplement à la turbine, landis que sur l'autre esi monlée l'excitatrice. L'arbre porte la rouc polaire qui est presséc hydaluliquement et clavetée sur celui-ci; il porte égalemenl les bagues collectrices en acier forgé. Ces dernières sont frettés a chaud sur l'arbre el isolées de celui-ci par du mica pur.

Les épanouissements polaires, et en partie aussi, les noyaux polaires sont lamellés et formés de tòles. Dans le but dobienir une courbe aussi sinusoïdale que possible, les épanouissements polaires sont placés obliquement el leurs arêtes convenablement arrondies. Les bobines inductrices sont constiluées par un ruban de cuivre enroulé de champ. Les spires onl été isolées les unes des autres par du carlon comprimé cl imprégné de gomme laque; loule la bobine a été ensuite soumisc à chaud à une forle pression lyydrauliçue, de façon à former ainsi un corps rigide et compact.

La construction dé l’allernateur a élé éludiée loul spécialement en vue dune ventilation efficace de loules les parties aclives. Des ailetles de ventilation à action puissanle sont fixces des deux côtés de la roùe polaire ; elles aspirent. l'air axialement et le refoulent en partic contre les lètes des bobines de l'induit el ell partie entre le pôles. Apres avoir balayé les bohines inductrices et l'enroulement induit, l'air traverse lo fer de l'induit par des canaux de ventilation largement dimensionnés el arrive à la carcasse d'où il s'échappe par les orifices de ventilation.

Le rhéostat de champ principal destiné à l'alternateur est exéculé avec plots de contact disposés sur un cadran circulaire.
Les résislances sont renfermées dans un châssis en fonte et en tôle perforée dans lequel l'air pour la ventilation accède facilement. Il se distingue avantageusement par sa construction solide.

L'avantage dans cet appareil consiste en ce que le nombre des positions de réglage est le double du nombre des plots de contact; le balai de contact, avant de quitter un plot, touche déjà le plot suivant; à ce moment-là deux résistances de même grandeur sont connectées en parallèle de sorte qu'entre chacune des positions de réglage correspondant à un plot, il se trouve une position intermédiaire et le nombre total des positions de réglage est ainsi doublé. Transformaleurs. - Les deux transformateurs correspondant
aux puissances des aux puissances des
alternateurs en fonction sont installés sur le premier étage en dessous de celui des alternateurs.

La place pour le troisième transformateur $y$ est aménagée.

Ces transformateurs à bain d'huile élèvent la tension de 220 des alternateurs à 15.000 volts.

Tableau de distribution. - Le tableau de distribution est du type pupitre et se trouve un peu surélevé dans la salle des machines vis-à-vis de l'alternateur médian, de façon que le mécanicien puisse manœuvrer aisément les appareils et surveiller

les trois groupes en mème temps.

Le pupitre a pour chaque groupe deux panneaux équipés porlant tous les appareils nécessaires, l'un affeclé à l'alternateur de 220 volts, l'autre aux lignes de départ de 15.000 volts.

\section{Conclusion}

Jepuis plus d'un an que le premier groupe des machines est en marche, tous les travaux en béton armé de l'usine-barrage se sont admirablement bien comportés.

L'aménagement intérieur de l'usine est disposé de telle façon que dans un espace relativement restreint, la surveillance, l'exploilation el l'entretien en général, se trouvent réduits à la plus simple expression.

La construction d'une hydro-électrique d'après cette conception devrait être employée plus fréquemmenl sur nos cours d'eau, car elle permet de faire de sensibles économies aussi bien au point de vue du prix de premier établissemenl quau point de vue des frais d'exploilation.

En comparaison avec un barrage plein en maçonnerie, le bar" rage en béton armé est en outre plus stable, et permet à chaque instant une visite facile de toutes ses parties. La preuve a été faite qu'un barrage construit d'après le type de Belle-Isle-enTerre peut être étanche dès la première mise sous l'eau. 